\title{
Coulomb energy differences in mirror nuclei revisited
}

\author{
B. K. Agrawal, ${ }^{1}$ Tapas Sil, ${ }^{1}$ S. K. Samaddar, ${ }^{1}$ J. N. De, ${ }^{2,3}$ and S. Shlomo ${ }^{3}$ \\ ${ }^{1}$ Saha Institute of Nuclear Physics, 1/AF Bidhannagar, Calcutta 700 064, India \\ ${ }^{2}$ Variable Energy Cyclotron Centre, 1/AF Bidhannagar, Calcutta 700 064, India \\ ${ }^{3}$ Cyclotron Institute, Texas A\&M University, College Station, Texas 77843
}

(Received 13 March 2001; published 29 June 2001)

\begin{abstract}
We calculate the Coulomb displacement energies (CDEs) of mirror nuclei using the recent parameter set (NL3) in the relativistic mean-field (RMF) model which includes self-coupling of the scalar meson. The results obtained are compared with the available ones calculated in the nonrelativistic Skyrme-Hartree-Fock (SHF) approach that have the best fit to the experimental data. When adjusted to reproduce the charge root-meansquare (rms) radius $r_{c}$ and the rms radii of the valence orbits, the results of the RMF model for the CDEs agree with those of the SHF model within $\sim 1 \%$. Our investigation also shows that, although the RMF with the NL3 parameter set reproduces the kink in the isotope variation of $r_{c}$, the values obtained for CDEs are too small to account for the experimental values without the addition of the contribution due to long-range correlation effects.
\end{abstract}

DOI: 10.1103/PhysRevC.64.024305

PACS number(s): PACS: 21.10.Sf, 21.60.-n

Coulomb displacement energies (CDEs) of analogue states (mirror nuclei), $\Delta E_{C}$, provide a stringent test for nuclear models. Nolen and Schiffer pointed out for the first time $[1,2]$ that a calculation of $\Delta E_{C}$ within the framework of the independent particle model (mean-field approach) leads to disagreement with experimental values. Using a WoodsSaxon potential well which reproduced the experimental charge distribution, Nolen and Schiffer (NS) found $\Delta E_{C}$ to be $\sim 7 \%$ smaller than the experimental values. It was also pointed out that attempts to remove this discrepancy in $\Delta E_{C}$ by adjusting the parameters of the potential well leads to surprisingly smaller values of the charge radii. This discrepancy between the experimental and theoretical evaluations of $\Delta E_{C}$ in mirror nuclei, referred to as the NS anomaly, has been the subject of many investigations in which various correction terms, namely, the exchange term, vacuum polarization, electromagnetic spin-orbit interaction, protonneutron mass difference, finite size effect of the proton, center of mass motion, Auerbach-Kahana-Weneser (AKW) effect (isospin impurity of the core), polarization of the core by the valence particle, and the Thomas-Ehrman effect (the difference between the neutron and proton wave functions), were considered $[1,3-10]$. It has been established that the net contribution of all these correction terms is too small to account for the anomaly $[4,11]$. The contribution of charge symmetry breaking (CSB) interaction was found $[4,11,12]$ to be $\sim 3 \%$ of $\Delta E_{C}$, i.e., accounting for only half of the discrepancy between theory and experiment.

It was first pointed out by Shlomo and Love [13] that, contrary to earlier estimates, the effect of long-range correlations (LRCs) on $\Delta E_{C}$ is not negligible. Using the particlevibration model and taking into account multipole excitations up to $L=5$, significant contributions of $\sim 1-3 \%$ to $\Delta E_{C}$ were obtained. Therefore, to explain the NS anomaly, it is necessary to go beyond the mean-field approximation and include the contributions due to the LRC effects and the CSB interaction; see also Refs. [14-16]. It is important to point out that long-range ground-state [random phase approximation (RPA)] correlation effects can account for the discrep- ancies between Skyrme-Hartree-Fock (SHF) predictions and experimental results for the charge root-mean-square (rms) radii $r_{c}$ associated with anomalous kinks in the mass dependence of $r_{c}$ (fluctuation in the isotope shift) $[17,18]$. Therefore a consistent description of $\Delta E_{C}$ and the anomalous kinks in $r_{c}$ is achieved by including the LRC contributions to the mean-field results obtained within the SHF approach.

In recent years, relativistic mean-field (RMF) [19] theory has been extremely successful in describing various facets of nuclear structure properties. The RMF theory with a small number of parameters is able to give a quantitative description of the ground-state properties [20-22] of spherical and deformed nuclei. Recently, a series of nonlinear RMF parameter sets has been proposed in order to explain the finer details of atomic nuclei. In particular, they have proved to be very successful in reproducing the anomalous kink in the isotope shift of $\mathrm{Pb}$ nuclei [23] and the first ever microscopic description of the anomalous isotopic shift in $\mathrm{Sr}$ and $\mathrm{Kr}$ chains [24]. The anomalous charge radii of $\mathrm{Cr}$ isotopes that required invocation of LRCs (zero-point oscillations) for an explanation in the nonrelativistic theory [17] also finds a natural explanation [25] in the RMF approach. Because of these successes of the RMF theory, in the present communication, we reexamine the problem of the Coulomb energy difference in mirror nuclei and calculate the CDEs within the RMF theory using recent parameter sets that proved successful in reproducing the anomalous kink in $r_{c}$. We also compare our results with earlier calculations performed in the nonrelativistic SHF formalism $[9,10]$ and in the RMF formalism [26].

The Coulomb energy difference in mirror nuclei is given by

$$
\Delta E_{C}=B(N+1, Z)-B(N, Z+1)
$$

where $B(N, Z)$ is the binding energy for a nucleus with $N$ neutrons and $Z$ protons. In this work the calculation is based 
on the self-consistent determination of the energy $E(A)$ of the core nucleus with $A$ nucleons $(N=Z)$ and then determining the energy difference

$$
\Delta=\mathcal{E}_{p}-\mathcal{E}_{n},
$$

where $\mathcal{E}_{p}$ and $\mathcal{E}_{n}$ are the single-particle energies of the proton and the neutron that have to be added to or removed from the core. The energy $\mathcal{E}$ (say, of a particle state) according to Koopman's theorem [27] satisfies the relation

$$
\mathcal{E}=E(A+1)-E(A) .
$$

Note that $\Delta$ forms the major part of $\Delta E_{c}$. As a result of the self-consistent calculation of $E(A), \Delta$ includes the contributions of the AKW and the Thomas-Ehrman effects [9]. A number of small but significant contributions like the exchange term, vacuum polarization, electromagnetic spinorbit interaction, proton-neutron mass difference, finite size effect of the proton, center of mass motion, and polarization of the core by the valence particle are to be added to $\Delta$ in order to compare it with $\Delta E_{c}$. Suzuki et al. [10] included these correction terms to the SHF evaluation of $\Delta$ with the parameter set SGII. With the inclusion of the contributions due to CSB interaction, they found that the theoretical values are smaller than the experimental one by $\sim 2 \%$. Marcos et al. [26] carried out RMF calculation of $\Delta E_{c}$, taking $\Delta$ from the SHF evaluation as a reference model for comparison with predictions from the RMF theory. Their results (with parameter set labeled R2) underestimate the SHF values considerably, typically by $\sim 5 \%$.

We perform calculations with the NL3 parameter set $[25,28]$ that includes nonlinear self-coupling of the $\sigma$ meson. We have also repeated calculations with the NL-SV2 parameter set [29]; here the self-coupling of vector meson [30] is further included. It has been shown [29] that vector selfcoupling might be important for an accurate description of nuclear shell effects, particularly for nuclei near the drip line.

We have carried out relativistic Hartree mean-field calculations for six pairs of mirror nuclei, with ${ }^{16} \mathrm{O},{ }^{40} \mathrm{Ca}$, and ${ }^{56} \mathrm{Ni}$ as the core. The Lagrangian density used to obtain the RMF equations is given as

$$
\begin{aligned}
\mathcal{L}= & \bar{\psi}\left(i \gamma^{\mu} \partial_{\mu}-M\right) \psi+\frac{1}{2} \partial^{\mu} \sigma \partial_{\mu} \sigma-U(\sigma)-g_{\sigma} \bar{\psi} \sigma \psi \\
& -\frac{1}{4} \Omega^{\mu \nu} \Omega_{\mu \nu}+\frac{1}{2} m_{\omega}^{2} \omega^{\mu} \omega_{\mu}-g_{\omega} \bar{\psi} \gamma^{\mu} \omega_{\mu} \psi-\frac{1}{4} \vec{R}^{\mu \nu} \vec{R}_{\mu \nu} \\
& +\frac{1}{2} m_{\rho}^{2} \vec{\rho}^{\mu} \vec{\rho}_{\mu}-g_{\rho} \bar{\psi} \gamma^{\mu} \vec{\rho}_{\mu} \vec{\tau} \psi-\frac{1}{4} F^{\mu \nu} F_{\mu \nu} \\
& -e \bar{\psi} \gamma^{\mu} \frac{\left(1-\tau_{3}\right)}{2} A_{\mu} \psi+\frac{1}{4} g_{4}\left(\omega_{\mu} \omega^{\mu}\right)^{2}
\end{aligned}
$$

which contains nucleons $(\psi)$ with mass $M, \sigma, \omega$, and $\rho$ mesons, the electromagnetic field, and nonlinear selfinteractions of the $\sigma$ field:
TABLE I. Comparison of the calculated values of charge radii (in fm) with the experimental data.

\begin{tabular}{lccccc}
\hline \hline Nucleus & NL3 & NL-SV2 & R2 $^{\text {a }}$ & SGII $^{\mathrm{a}}$ & Expt. $^{\mathrm{b}}$ \\
\hline${ }^{16} \mathrm{O}$ & 2.674 & 2.684 & 2.75 & 2.75 & 2.693 \\
${ }^{32} \mathrm{~S}$ & 3.282 & 3.291 & - & - & 3.263 \\
${ }^{40} \mathrm{Ca}$ & 3.469 & 3.438 & 3.48 & 3.47 & 3.478 \\
${ }^{48} \mathrm{Ca}$ & 3.471 & 3.456 & 3.48 & - & 3.479 \\
${ }^{56} \mathrm{Ni}$ & 3.709 & 3.716 & - & - & $3.73^{\mathrm{c}}$ \\
${ }^{90} \mathrm{Zr}$ & 4.269 & 4.254 & 4.27 & - & 4.270 \\
${ }^{208} \mathrm{~Pb}$ & 5.513 & 5.513 & 5.53 & - & 5.504 \\
\hline \hline
\end{tabular}

${ }^{a}$ Reference [26].

${ }^{\mathrm{b}}$ Reference [31].

${ }^{\mathrm{c}}$ An estimate; see text.

$$
U(\sigma)=\frac{1}{2} m_{\sigma}^{2} \sigma^{2}+\frac{1}{3} g_{2} \sigma^{3}+\frac{1}{4} g_{3} \sigma^{4}
$$

For the NL3 parameter set, the last term in Eq. (4) is absent. The third component of isospin $\tau_{3}$ equals -1 for protons and 1 for neutrons. Recourse to the variational principle followed by the mean-field approximation treating the fields as $c$ numbers results in the Dirac equation for the nucleon and KleinGordon-type equations for the mesons and the photon (see Ref. [21] for details).

The value of $\Delta E_{C}$ is determined predominantly by the rms value of the charge distribution of the core and that of the valence proton, in a particle or hole state. To facilitate the discussions, in Table I we present the calculated charge radii for a host of spherical nuclei over the periodic table and compare them with the experimental data [31,32]. Since the charge rms radius $r_{c}$ of ${ }^{56} \mathrm{Ni}$ is not known, we assess its value using the experimental values of ${ }^{58} \mathrm{Ni},{ }^{56} \mathrm{Fe}$, and ${ }^{54} \mathrm{Fe}$ [31]. We take $r_{c}\left({ }^{56} \mathrm{Ni}\right)=r_{c}\left({ }^{58} \mathrm{Ni}\right)-\left[r_{c}\left({ }^{56} \mathrm{Fe}\right)-r_{c}\left({ }^{54} \mathrm{Fe}\right)\right]$ $=3.73 \mathrm{fm}$, assuming isotope shifts due to the two $2 p_{3 / 2}$ neutrons in the $\mathrm{Ni}$ isotopes and in the $\mathrm{Fe}$ isotopes to be the same. The charge radii are reproduced to within $\sim 0.5 \%$ accuracy with the NL3 set and $\sim 1 \%$ accuracy with the NL-SV2 set. The R2 parameter set also explains the experimental data extremely well. The SHF calculation for the nuclei ${ }^{16} \mathrm{O}$ and ${ }^{40} \mathrm{Ca}$ with the SGII parameter set [10] also reproduces their experimental charge radii reasonably.

TABLE II. The RMF and Skyrme-Hartree-Fock results for $\Delta$ $=\mathcal{E}_{p}-\mathcal{E}_{n}$ and the experimental Coulomb energy differences $\Delta E_{C}$ in mirror nuclei $(\mathrm{MeV})$.

\begin{tabular}{lccccc}
\hline \hline System & NL3 & NL-SV2 & R2 $^{\text {a }}$ & SGII $^{\text {a }}$ & $\Delta E_{C}$ (expt. $\left.{ }^{\mathrm{b}}\right)$ \\
\hline${ }^{15} \mathrm{O}-{ }^{15} \mathrm{~N}$ & 3.925 & 3.876 & 3.667 & 3.808 & 3.536 \\
${ }^{17} \mathrm{~F}^{17} \mathrm{O}$ & 3.575 & 3.525 & 3.290 & 3.665 & 3.543 \\
${ }^{39} \mathrm{Ca}-{ }^{39} \mathrm{~K}$ & 7.352 & 7.381 & 7.249 & 7.450 & 7.313 \\
${ }^{41} \mathrm{Sc}-{ }^{41} \mathrm{Ca}$ & 7.011 & 7.090 & 6.868 & 7.289 & 7.278 \\
${ }^{55} \mathrm{Ni}^{55} \mathrm{Co}$ & 9.504 & 9.500 & - & - & 9.422 \\
${ }^{57} \mathrm{Cu}-{ }^{57} \mathrm{Ni}$ & 8.895 & 9.376 & - & - & 9.499 \\
\hline \hline
\end{tabular}

${ }^{\mathrm{a}}$ Reference [26].

${ }^{\mathrm{b}}$ Reference [35]. 
TABLE III. Comparison of the calculated values of the rms radii (in fm) for the valence neutron orbitals with the experimental data.

\begin{tabular}{lcccc}
\hline \hline Nucleus & NL3 & NL-SV2 & SGII $^{\text {a }}$ & Expt. $^{\text {b }}$ \\
\hline${ }^{15} \mathrm{O}$ & 2.517 & 2.814 & 2.825 & - \\
${ }^{17} \mathrm{O}$ & 3.482 & 3.408 & 3.275 & 3.36 \\
${ }^{39} \mathrm{Ca}$ & 3.689 & 3.718 & 3.606 & - \\
${ }^{41} \mathrm{Ca}$ & 4.156 & 4.105 & 3.989 & 3.99 \\
\hline \hline
\end{tabular}

${ }^{\text {a Reference [26]. }}$

${ }^{b}$ References [33,34].

In Table II, the values of the Coulomb energy difference $\Delta=\mathcal{E}_{p}-\mathcal{E}_{n}$, calculated in our model, are compared with those from the R2 set and also from the SGII set; as explained earlier, the results from the latter set are taken to be a good reference for comparison. In the earlier RMF calculation with the R2 parameter set, it is seen that the discrepancy with the SGII results is $\sim 5 \%$. With the NL3 parameter set, the said discrepancy is reduced to $\sim 2.5 \%$. Thus one can find that there is an overall improvement by approximately a factor of 2 with the NL3 parameter set. One also notes that with the inclusion of self-coupling of vector mesons (NL$\mathrm{SV} 2$ set), except for the $\mathrm{Cu}-\mathrm{Ni}$ pair, the results for $\Delta$ are only marginally changed.

Information on the valence neutron and proton wave functions can be deduced from the magnetic form factor obtained in backward electron scattering. Since their extension has a seminal role in the determination of the Coulomb energy, their calculations and comparison with the available experimental data may throw more light on the intricate nature of the Coulomb energy anomaly. In Table III, results for the radii of the valence neutron orbits are presented for the $\mathrm{O}$ and $\mathrm{Ca}$ isotopes. With the NL3 parameter set, the rms radii of the valence neutron orbitals in ${ }^{17} \mathrm{O}$ and ${ }^{41} \mathrm{Ca}$ are larger than the experimental values $[33,34]$ by $\sim 4 \%$, leading to a decrease of $\Delta$ by $\sim 2 \%$. Therefore, increasing the values of $\Delta$ for the $A=17$ and 41 mirror nuclei obtained in the RMF calculation with the NL3 parameter set by $\sim 2 \%$, we obtain an agreement within $\sim 1 \%$ with the results obtained with the SGII interaction; see Table II. The net contributions $[4,10]$ due to the exchange term, vacuum polarization, electromagnetic spin-orbit interaction, proton-neutron mass difference, finite size effect of the proton, center of mass motion, and polarization of the core by the valence particle decrease the calculated CDEs by about $0.45,0.20,0.40$, and $0.30 \mathrm{MeV}$ for the $A=15,17,39$, and 41 mirror nuclei, respectively. Thus, the discrepancy between the mean-field (SHF or RMF) results for the CDEs and the corresponding experimental values is reduced to about $3-5 \%$. As pointed out earlier, this remaining discrepancy can be accounted for by including the contributions due to CSB interaction and LRC effects [13].

To conclude, we have calculated the Coulomb energy differences in some pairs of mirror nuclei with recent nonlinear parameter sets (NL3 and NL-SV2) in the relativistic meanfield theory. These results are compared to nonrelativistic Skyrme-Hartree-Fock calculations with the SGII interaction and also with the earlier RMF results (R2). Compared to the calculations with the $\mathrm{R} 2$ parameter set, the present results are in closer agreement with the SGII values. Inclusion of the self-coupling of the vector meson improves the results further. When adjusted to reproduce $r_{c}$ and the rms radius of the valence orbits, the RMF model (with the NL3 parameter set) results for $\Delta$ agree with those of the SHF model within $\sim 1 \%$. An agreement with experimental data is obtained by adding the contributions due to the CSB interaction and LRC effects. Our investigation also shows that although the RMF theory with the NL3 parameter set reproduces the kink in the isotope variation of $r_{c}$, the values obtained for $\Delta$ are too small to account for the experimental values of the CDEs without the addition of the contribution due to the LRC effects.

J.N.D. thanks the Cyclotron Institute at Texas A\&M University for kind hospitality. S.S. thanks the variable Energy Cyclotron Centre, Calcutta for kind hospitality. This work was supported in part by the U.S. Department of Energy under Grant No. DOE-FG03-93ER40773.
[1] J. A. Nolen and J. P. Schiffer, Annu. Rev. Nucl. Sci. 19, 471 (1969).

[2] J. A. Nolen and J. P. Schiffer, Phys. Lett. 29B, 396 (1969).

[3] K. Okamoto, Phys. Lett. 11, 150 (1964).

[4] S. Shlomo, Rep. Prog. Phys. 41, 957 (1978), and references therein.

[5] N. Auerbach, Phys. Rep. 98, 274 (1983).

[6] E. H. Auerbach, S. Kahana, and J. Weneser, Phys. Rev. Lett. 23, 1253 (1969).

[7] C. W. Wong, Nucl. Phys. A151, 323 (1970).

[8] J. Damgaard, C. K. Scott, and E. Osnes, Nucl. Phys. A154, 12 (1969).

[9] N. Van Giai, D. Vautherin, M. Veneroni, and D. M. Brink, Phys. Lett. 35B, 135 (1971).

[10] T. Suzuki, H. Sagawa, and A. Arima, Nucl. Phys. A536, 141 (1992).
[11] S. Shlomo, Phys. Lett. 42B, 146 (1972).

[12] R. Machleidt and H. Muther, Phys. Rev. C 63, 034005 (2001).

[13] S. Shlomo and W. G. Love, Phys. Scr. 26, 280 (1982).

[14] V. R. Shaginyan, Yad. Fiz. 39, 346 (1984) [Sov. J. Nucl. Phys. 39, 218 (1984)].

[15] A. Bulgac and V. R. Shaginyan, Phys. Lett. B 469, 1 (1999).

[16] S. A. Fayans, Pis'ma Zh. Éksp. Teor. Fiz. 68, 161 (1998) [JETP Lett. 68, 169 (1998)].

[17] S. Shlomo, Phys. Lett. B 209, 23 (1988).

[18] F. Baranco and R. A. Broglia, Phys. Lett. 151B, 90 (1985).

[19] B. D. Serot and J. D. Walecka, Adv. Nucl. Phys. 16, 1 (1986).

[20] A. Bouyssy, S. Marcos, and P. Van Thien, Nucl. Phys. A422, 541 (1984).

[21] Y. K. Gambhir, P. Ring, and A. Thimet, Ann. Phys. (N.Y.) 198, 132 (1990).

[22] P. Ring, Prog. Part. Nucl. Phys. 37, 193 (1996). 
[23] M. M. Sharma, G. A. Lalazissis, and P. Ring, Phys. Lett. B 317, 9 (1993).

[24] G. A. Lalazissis and M. M. Sharma, Nucl. Phys. A586, 201 (1995).

[25] G. A. Lalazissis, S. Raman, and P. Ring, At. Data Nucl. Data Tables 71, 1 (1999).

[26] S. Marcos, N. Van Giai, and L. N. Savaskin, Nucl. Phys. A549, 143 (1992).

[27] T. Koopman, Physica (Amsterdam) 1, 104 (1934).

[28] G. A. Lalazissis, J. Konig, and P. Ring, Phys. Rev. C 55, 540 (1997).
[29] M. M. Sharma, A. R. Farhan, and S. Mythili, Phys. Rev. C 61, 054306 (2000).

[30] A. R. Bodmer, Nucl. Phys. A526, 703 (1991).

[31] G. Fricke et al., At. Data Nucl. Data Tables 60, 177 (1995).

[32] H. de Vries, C. W. de Jagar, and C. de Vries, At. Data Nucl. Data Tables 36, 495 (1987).

[33] N. Kalantar-Nayestanaki et al., Phys. Rev. Lett. 60, 1707 (1988).

[34] S. Platchkov et al., Phys. Rev. Lett. 61, 1465 (1988).

[35] G. Audi, O. Bersillon, J. Blanchot, and A. H. Wapstra, Nucl. Phys. A624, 1 (1997). 